\title{
TAIGA-HiSCORE: results from the first two operation seasons
}

M. Tluczykont ${ }^{6}$, O. Gress ${ }^{2}$, E. Korosteleva ${ }^{1}$, L. Kuzmichev ${ }^{1,2}$, A. Pakhorukov ${ }^{2}$,

A. Porelli $^{8}$, V. Prosin ${ }^{1}$, L. Sveshnikova ${ }^{1}$, R. Wischnewski ${ }^{8}$, I. Astapov ${ }^{9}$,

P. Bezyazeekov ${ }^{2}$, V. Boreyko ${ }^{10}$, A. Borodin ${ }^{10}$, M. Brueckner ${ }^{8}$, N. Budnev ${ }^{2}$,

A. Chiavassa ${ }^{4}$, A. Dyachok ${ }^{2}$, O. Fedorov ${ }^{2}$, A. Gafarov ${ }^{2}$, A. Garmash ${ }^{11}$,

N. Gorbunov ${ }^{10,14}$, V. Grebenyuk ${ }^{10,14}$, T. Gress ${ }^{2}$, O. Grishin ${ }^{2}$, A. Grinyuk $^{10}$, D. Horns ${ }^{6}$,

A. Ivanova ${ }^{2}$, N. Kalmykov ${ }^{1}$, Y. Kazarina ${ }^{2}$, V. Kindin ${ }^{9}$, P. Kirilenko ${ }^{11}$, S. Kiryuhin ${ }^{2}$,

R. Kokoulin ${ }^{9}$, K. Kompaniets ${ }^{9}$, V. Kozhin ${ }^{1}$, E. Kravchenko ${ }^{11,12}$, M. Kunnas ${ }^{6}$,

Yu. Lemeshev ${ }^{2}$, V. Lenok ${ }^{2}$, B. Lubsandorzhiev ${ }^{1,3}$, N. Lubsandorzhiev ${ }^{1}$, R. Mirgazov ${ }^{2}$,

R. Mirzoyan ${ }^{5,2}$, R. Monkhoev ${ }^{2}$, R. Nachtigall ${ }^{6}$, E. Osipova ${ }^{2}$, M. Panasyuk ${ }^{1}$,

L. Pankov ${ }^{2}$, A. Petrukhin ${ }^{9}$, V. Poleschuk ${ }^{2}$, E. Popescu ${ }^{13}$, E. Popova ${ }^{1}$, E. Postnikov ${ }^{1}$,

V. Ptuskin ${ }^{7}$, E. Rjabov' ${ }^{2}$, G. Rubtsov ${ }^{3}$, A. Pushnin ${ }^{2}$, Y. Sagan ${ }^{10}$, B. Sabirov ${ }^{10}$,

V. Samoliga ${ }^{2}$, Yu. Semeney ${ }^{2}$, A. Silaev ${ }^{1}$, A. Silaev(junior) ${ }^{1}$, A. Sidorenkov ${ }^{3}$,

A. Skurikhin ${ }^{1}$, V. Slunecka ${ }^{10}$, A. Sokolov ${ }^{11,12}$, C. Spiering ${ }^{8}$, V. Tabolenko ${ }^{2}$,

B. Tarashansky ${ }^{2}$, A. Tkachenko ${ }^{10}$, L. Tkachev ${ }^{10,14}$, A. Zagorodnikov ${ }^{2}$, D. Zhurov ${ }^{2}$, V. Zurbanov ${ }^{2}$, I. Yashin*9 (TAIGA collaboration)

${ }^{1}$ Skobeltsyn Institute of Nuclear Physics MSU, Moscow, Russia

${ }^{2}$ Institute of Applied Physics ISU, Irkutsk, Russia, ${ }^{3}$ Institute for Nuclear Research of RAN, Moscow, Russia, ${ }^{4}$ Dipartimento di Fisica Generale Universiteta di Torino and INFN, Torino, Italy, ${ }^{5}$ Max-Planck-Institute for Physics, Munich, Germany, ${ }^{6}$ Institute for Experimental Physics, University of Hamburg, Germany, ${ }^{7}$ IZMIRAN, Moscow, Russia, ${ }^{8}$ DESY, Zeuthen, Germany,

${ }^{9}$ National Research Nuclear University MEPhI (Moscow Engineering Physics Institute), Moscow, Russia, ${ }^{10}$ JINR, Dubna, Russia, ${ }^{11}$ Novosibirsk State University, NSU, Novosibirsk, Russia, ${ }^{12}$ Budker Institute of Nuclear Physics SB RAS, Novosibirsk, Russia, ${ }^{13}$ ISS, Bucharest, Romania ${ }^{14}$ Dubna State University, Dubna, Russia E-mail:

martin.tluczykontephysik.uni-hamburg.de

The very to ultra high energy gamma-ray regime up to several $100 \mathrm{TeV}$ is the key to spectrally resolve the cutoff regime of the long-sought Pevatrons, the Galactic cosmic-ray PeV accelerators. One component of the TAIGA hybrid detector is the TAIGA-HiSCORE shower-front sampling timing array, which currently consists of 28 wide angle $(0.6 \mathrm{sr})$ air Cherenkov timing stations distributed on an area of $0.25 \mathrm{~km}^{2}$, and which will be doubled in area by fall 2017 . We report on the status and first results of the HiSCORE timing array after the first two operation seasons, including calibration studies on timing and pointing, cross calibration with data from the first TAIGA-IACT, observations with the timing array of a LIDAR operating on the ISS, a measurement of the Cosmic Ray spectrum, and a search for high energy gamma-rays from Galactic sources such as the Crab Nebula.

35th International Cosmic Ray Conference - ICRC2017

10-20 July, 2017

Bexco, Busan, Korea 


\section{Introduction}

More than 150 sources of very high energy $(\mathrm{E}>100 \mathrm{GeV})$ gamma rays [1] are known today. The ultra high energy (UHE, E $>10 \mathrm{TeV}$ ) gamma ray regime is still only poorly covered. Given the quick drop of the flux of astrophysical sources with increasing energy, very large instrumented areas are required to access this regime. A cost effective method to realize large areas and a wide field of view is the shower-front timing technique (also: shower-front sampling). The weak point of such a non-imaging technique is a poor gamma-hadron separation at low energies. This can be compensated by a hybrid combination with a small number of small sized imaging air Cherenkov telescopes (IACTs), such as currently constructed by the TAIGA (Tunka Advanced Instrument for Gamma ray and cosmic-ray Astrophysics) experiment [18, 3, 4]. The hybrid approach combines the timing and imaging techniques using a new hybrid reconstruction (see e.g. [12, ] and [13, 14]).

The main astrophysical motivation of TAIGA is the spectroscopic measurement of the cutoff regime of known Galactic sources. Some of these sources might be cosmic-ray accelerators reaching up to the knee energies (around $3 \mathrm{PeV}$ proton energy), i.e. the long-sought Pevatrons. More astrophysical motivations are given in [5, 6]. Here, we present the status and first results of the timing detector component of TAIGA: HiSCORE (High Sensitivity Cosmic ORigin Explorer).

\section{The HiSCORE detector component of TAIGA}

\subsection{Detector layout}

The TAIGA-HiSCORE timing array currently consists of 28 timing stations distributed over an area of $0.25 \mathrm{~km}^{2}$ in the Tunka valley in Siberia $\left(51^{\circ} 48^{\prime} 47.5^{\prime \prime} \mathrm{N}, 103^{\circ} 04^{\prime} 16.3^{\prime \prime} \mathrm{E}, 675 \mathrm{~m}\right.$ a.s.1.). The distance between stations ranges from $75 \mathrm{~m}$ to $150 \mathrm{~m}$ (see Fig. 1). Ongoing deployment (until October 2017) will extend the array to a total of 58 stations on $0.6 \mathrm{~km}^{2}$. This array is an implementation of the HiSCORE wide-angle timing array concept [7]. A TAIGA-HiSCORE station consists of four 8 inch-photomultiplier tubes (PMTs), each equipped with light a concentrator (Winston cone), resulting in $0.5 \mathrm{~m}^{2}$ light collection area, and a field of view of $0.6 \mathrm{sr}$ per detector station. The PMTs, voltage-divider, preamplifiers, and light concentrators are installed under a protective plexiglas cover with a heating-wire system (to prevent condensation) in a box with a sliding lid mechanism. All other electronics parts for local station DAQ and slow control are installed in a separate heated electronic box. The optical axis of the stations can be tilted in the north-south direction, therewith varying the part of the sky covered throughout the year. In the northern hemisphere, tilting to the north results in a smaller total sky area covered, but at deeper exposure (about $1000 \mathrm{~h}$ per year). Tilting to the south increases the coverage at the expense of exposure per square-degree. During the first two operation seasons, the stations were tilted to the south, allowing observations of the Crab Nebula. In addition to the timing array, a first small IACT (4.75 $\mathrm{m}$ dish diameter) was built in 2016 and has taken first data in the last observation season. The IACT detector part is addressed in an overview of the TAIGA experiment in [18] and in [20].

\footnotetext{
* Speaker.
} 


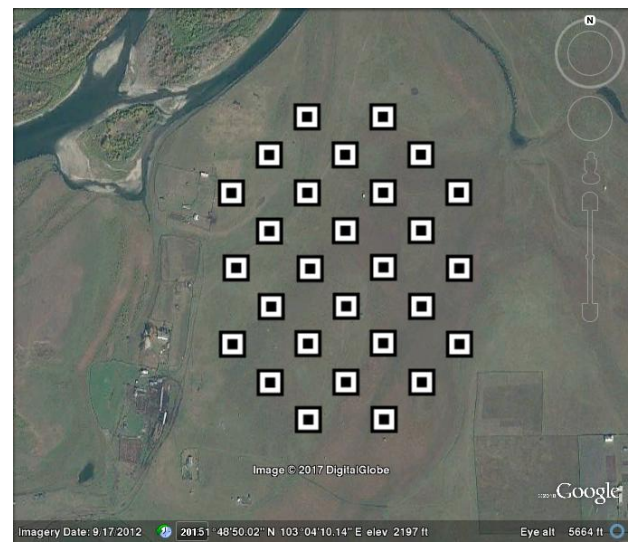

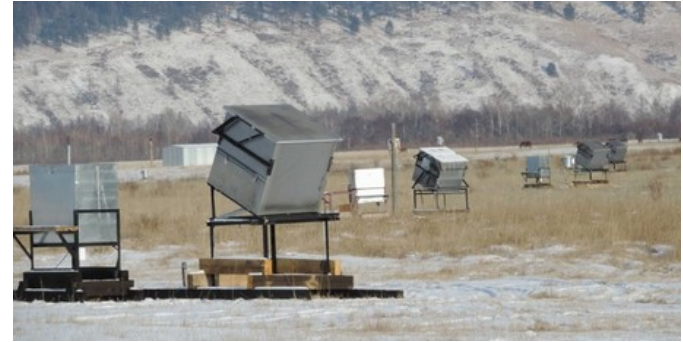

Figure 1: Left: The Layout of the 2017 HiSCORE TAIGA array (satellite picture by google-earth). Right: 3 HiSCORE stations with their electronic boxes.

\subsection{Station DAQ}

The DAQ is based on fast $\mathrm{GHz}$ signal sampling and operates at a trigger rate of the order of $20 \mathrm{~Hz}$ [8]. The PMTs are operated in a high noise environment (night-sky background) at a gain of the order of $10^{4}$. The anode of each PMT is read out and sampled at $2 \mathrm{GHz}$ with a DRS 4-based readout board. An analog summator board is used to split the anode signals. If the analog sum of the anode signals is in excess of a programmable threshold value, a trigger is issued and the anode signals are sampled with the DRS 4 chip. In order to increase the dynamic range for the signal amplitude, also the 5th dynodes are read out. The high-gain channel (anodes) is amplified with a factor of 30, and the low-gain channel (dynodes) with a factor 4. The 9th channel of the DRS 4-based readout board is used for sampling of a central $100 \mathrm{MHz}$ clock which is distributed over optical fiber from the central DAQ. The clock is used by a custom-made time synchronization system and combined with an ethernet-based WhiteRabbit system, providing a central GPS-disciplined Rubidium clock [18]. Upon local trigger, station data are transferred via optical fiber to the central DAQ. Event building is processed offline using the sub-ns precision time-stamps.

\subsection{Performance, and reconstruction quality}

Estimations of the sensitivity of TAIGA [15], based on previous simulations [7, 13, 11], result in a sensitivity at the level of $2.5 \cdot 10^{-13} \mathrm{TeV} \mathrm{cm}^{-2} \mathrm{~s}^{-1}$ at $100 \mathrm{TeV}$ for a hybrid timing-imaging-array with an instrumented area of $1 \mathrm{~km}^{2}$ and 3 IACTs (funded and planned for 2019) [18].

The energy threshold of the current HiSCORE array is estimated to be $50 \mathrm{TeV}$ for gamma rays. This value was obtained using a full detector simulation, reproducing the observed trigger rates with simulated hadronic air showers. The observed rates could be reproduced using a station threshold of 250p.e., resulting in a differential gamma ray spectrum with a maximum at $50 \mathrm{TeV}$. The same result was obtained using an independent approach, based on the normalization of the observed array trigger rate to the known flux of cosmic rays (see Figure 2). In an earlier stage of the experiment, these methods were cross-checked by measurements and simulations of the trigger rate as a function of the discriminator threshold. The found value of the threshold is currently dictaded by the chosen station discriminator thresholds that limit the station trigger rate to $20 \mathrm{~Hz}$. A faster local station readout system in combination with online air shower event filtering/building can further reduce this energy threshold. In a future stage of the experiment also a higher altitude site might be envisaged, allowing a lower energy threshold at the cost of a higher station density. 


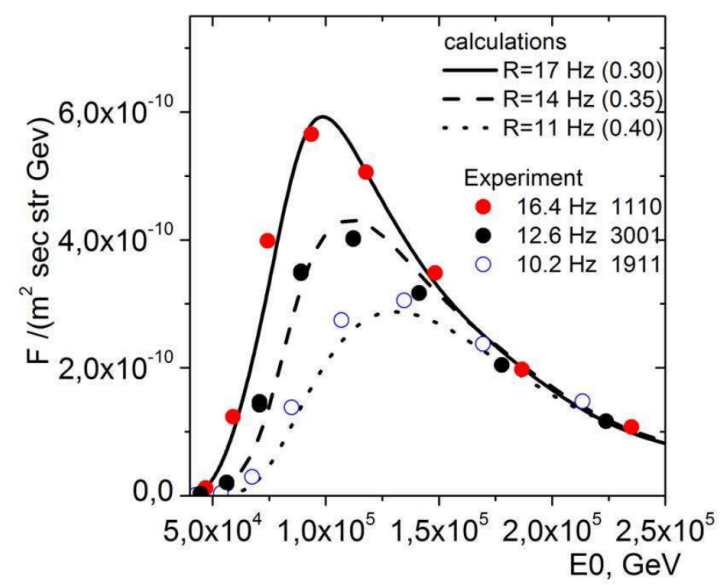

Figure 2: The differential energy threshold for cosmic rays obtained from data (points) for different observation nights of the operation season 2015/16 and simulations (lines). From this plot, the energy threshold for cosmic rays (mainly protons) can be deduced. The energy threshold for gamma rays is lower due to higher light yield of electromagnetic air showers.

\subsection{Event reconstruction}

Parameters used for reconstruction are the signal amplitude, charge, and half-rise time, using methods developed for Tunka-133 and HiSCORE [10, 11, 9]. The shower core is first reconstructed with a simple center-of-gravity method, and then refined using a fit of station light densities to a lateral density function. Alternatively, a fit to the amplitude distribution function (ADF) can be performed. A first order direction is obtained from a fit of station arrival times to a plane, followed by more realistic arrival time models, such as a cone-fit, or an analytical model of the arrival times depending on the primary direction, shower core, and shower height. An event display for one reconstructed air shower event is shown in Figure 3. Shown are the core reconstruction using the ADF, as well as a arrival time fit for directional reconstruciton. The shower height is also deduced from the slopes of the LDF or ADF fits. As energy estimator, the value of the LDF at a certain distance to the shower core (typically $200 \mathrm{~m}$ ) is used.
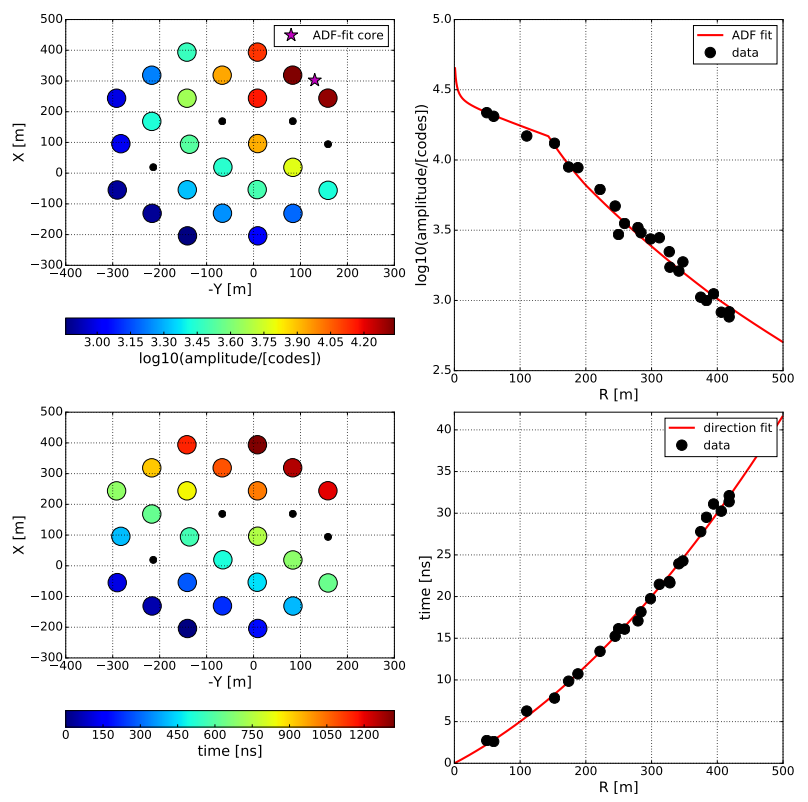

Figure 3: Event display of an air shower event (real data). Top, left: distribution of amplitudes over the array (amplitudes are only shown for stations used in the reconstruction of this event). The position of the air shower core impact position obtained from the fit is indicated with a star. Top, right: Amplitude distribution function vs. core impact distance. Bottom: distribution of station arrival times relative to the shower plane and the direction fit to the station arrival times as a function of core distance. 
The reconstruction quality was investigated using comparisons of Monte Carlo Simulations to real data. The chessboard method was used to verify our understanding of the detector performance in Monte Carlo simulations [15]. As shown in Figure 4, the angular resolution of the HiSCORE detector approaches $0.1^{\circ}$ at high station multiplicities. The energy resolution is of the order of

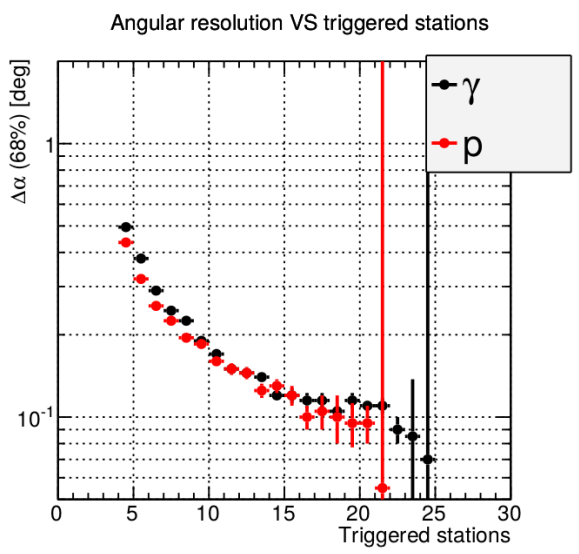

Figure 4: The angular resolution of TAIGAHiSCORE as a function of the station multiplicity, i.e. the number of stations in the event. This dependence was obtained from simulations of the 28-station array. The Monte Carlo simulations were previously validated using real data (see text).

typical values for air Cherenkov experiments, reaching $10 \%$ at high energies.

\subsection{Time calibration}

Previously, it was shown that a station time jitter of better than $1 \mathrm{~ns}(\mathrm{rms})$ is required in order to reach optimum angular resolution [11]. Two independent station time synchronization methods were successfully employed, and shown to meet these requirements $[9,8,18]$.

As opposed to this $1 \mathrm{~ns}$ station clock stability, relevant for the point-spread function of the detector, the constant relative time-delays between detector stations, or time-offsets $\Delta t_{o f f, i}$ (electronics/sensor/cable delays etc.) must also be known with ns-precision, since they may introduce a systematic mis-pointing of the array. Several independent methods, using external light sources of known location, have been developed to determine $\Delta t_{o f f, i}$.

- LED-calibration: a nsec-pulsed LED emits light horizontally over the array. Diffuse light reflectors deflect the light into a subset of HiSCORE stations. The time offsets $\Delta t_{o f f, i}$ are derived from measured arrival times and geometry (see e.g. for HiSCORE-9, [9]).

- Combined EAS- and LED-calibration: EAS events can be used to obtain an approximate time-offset calibration, by iterating the offsets $\Delta t_{o f f, i}$ to minimize the $\chi^{2}$ when reconstructing the EAS shower front (for high-multiplicity events). As shown in [17], the inherent mispointing of this approach (which is small if all initial offsets are only a few ns), can be corrected by adding a few LED-determined time-offsets ("Hybrid method").

- ISS-calibration: the regularly recorded CATS-LIDAR events from the ISS (see Section 2.6, and [16]) provide, for clear weather conditions, a calibration beam, similar to an LED placed at extremely large distance. The $\Delta t_{o f f, i}$ can be derived from the measured arrival times and the known location of the ISS (i.e. the laser). An advantage of the method is the instantaneous illumination of the full array. 
The results of the different time offset calibrations performed for one run are shown in Figure 5. The deviations between the calibration methods are shown on the left side. The distributions of the time delays over the array is shown on the right side, amounting to only few ns (rms).
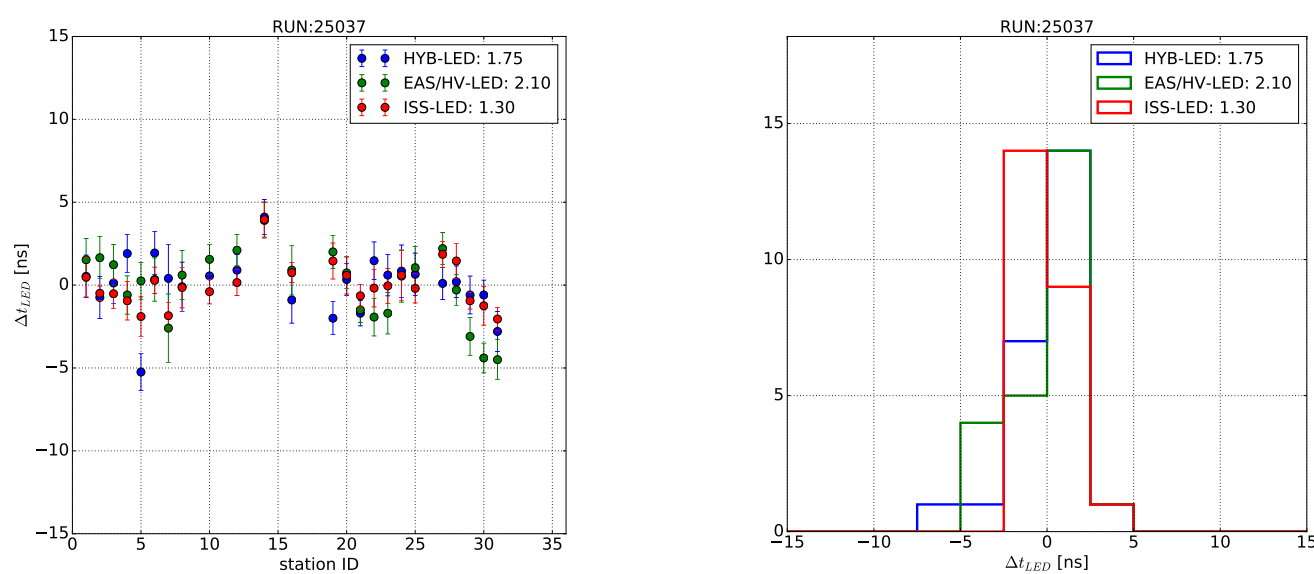

Figure 5: Resulting absolute time offsets $\Delta t_{o f f, i}$ per station $i$. The calibration results are shown for the different procedures described above for one run. Left: Deviations between methods. Right: Distribution of the deviations of all stations over the array.

\subsection{First results}

Background study and Crab Nebula data: Applying a standard ring background method on a large test region of $12^{\circ} \times 12^{\circ}$ blinded real data, the field of view was found to be free of fluctuation artifacts in excess of what is statistically expected (see Figure 6). Performing the same analysis (no optimization, no $\mathrm{g} / \mathrm{h}$ separation) on the position of the Crab Nebula, a hint of a signal is found [19, these proceedings]. While not significant, these observations are compatible with reasonable Crab Nebula flux extrapolations to higher energies. We expect to reach better sensitivity optimizing the
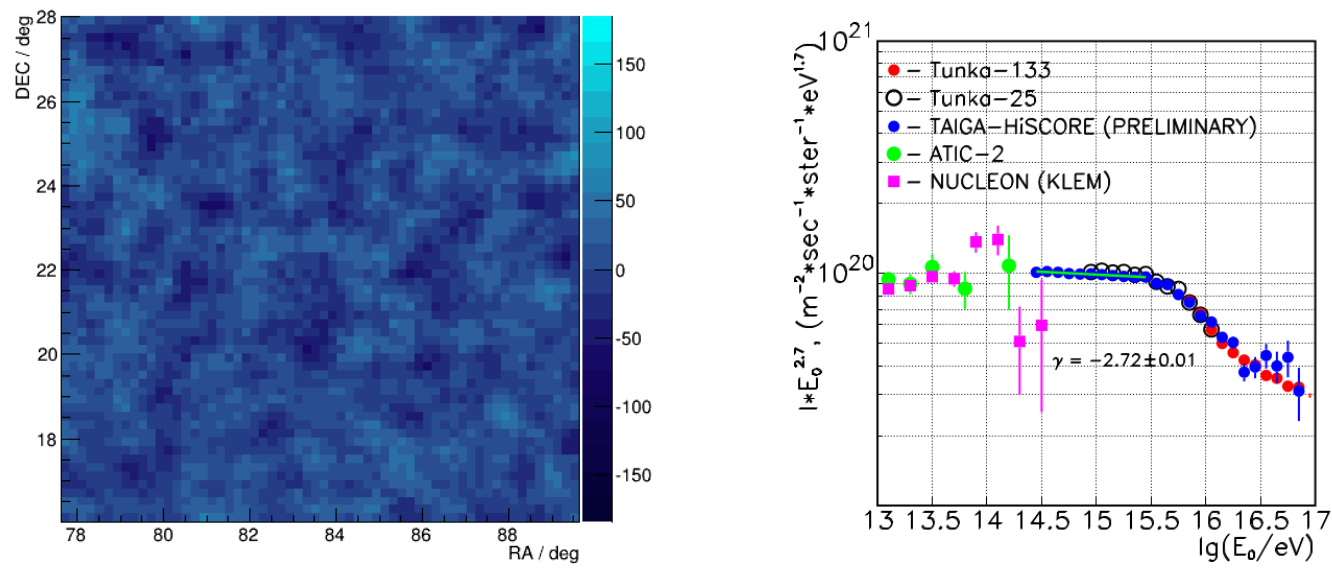

Figure 6: Left: Excess map of blinded real data using the ring background method. No significant excess is expected. In the shown 12 by 12 degree field of view, fluctuations agree with statistical variations, demonstrating the applicability of the ring background model over a wide field of view. Right: All-particle cosmic ray spectrum as observed by TAIGA-HiSCORE compared to results from other experiments. 
analysis, increasing the area as planned, and combining the timing array with IACTs.

The cosmic ray energy spectrum: Using the reconstruction methods developed for the Tunka133 cosmic ray array and for HiSCORE [10, 11], the energy spectrum of cosmic rays was reconstructed for the data of the HiSCORE-TAIGA array. As can be seen in Figure 6, the obtained all-particle spectrum is consistent with previous measurements.

The ISS CATS LIDAR: the serendipitous detection of the CATS LIDAR onboard the ISS is discussed in [16], and was previously reported in [15]. The LIDAR laser provides a short pulsed, powerful and highly focused $532 \mathrm{~nm}$ light beam, registered by HiSCORE as a plane wave. Eleven passages were detected during the past two observation seasons. Simultaneous observations of HiSCORE and the optical telescope MASTER-Tunka [22] allowed to verify that the absolute pointing of HiSCORE is better than $0.1^{\circ}$. These data are used for TAIGA timing calibration and for various precision performance checks. Possibly, also atmospheric light scattering studies between space and ground could be done with the same data.

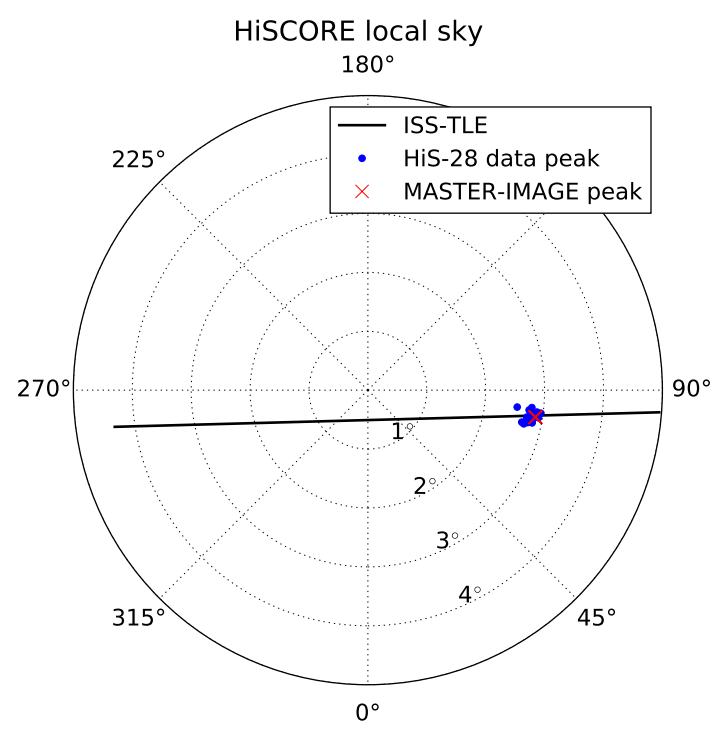

Figure 7: Reconstructed directions of CATS LIDAR events observed by HiSCORE (blue dots) during a passage of the ISS over the array. The orbital path of the ISS is superimposed to the image. An optical image from the MASTER telescope (peak shown as red cross) confirms the ISS path. The reconstructed HiSCORE directions were calculated using the Combined LED-EAS time-delay calibration.

\section{Summary}

The HiSCORE-TAIGA timing array is a part of the hybrid timing-imaging TAIGA experiment. Since October 2015, 28 timing stations distributed over an area of $0.25 \mathrm{~km}^{2}$ are being operated in the Tunka valley in Siberia. The total detector area is currently being upgraded to $0.6 \mathrm{~km}^{2}$ (30 additional stations). A first IACT is in commissioning since the last observation season. The hybrid array ( $1 \mathrm{~km}^{2}$, 3 IACTs) is expected to have a sensitivity of $2.5 \cdot 10^{-13} \mathrm{TeV} \mathrm{cm}^{-2} \mathrm{~s}^{-1}$ at $100 \mathrm{TeV}$ by 2019. Comparisons of timing array real data to Monte Carlo simulations show that the HiSCORE detector is reasonably well understood. While our Crab Nebula data show a hint to a signal, compatible with expectations based on UHE flux extrapolations. In the current stage, no significant excess is expected from the Crab Nebula. The CATS-LIDAR onboard the ISS (seredipitously detected) is now regularly used for precision time calibration and a verification of the absolute pointing of the experiment. 


\section{Acknowledgements}

This work was supported by the Russian Federation Ministry of Education and Science (projects 14.B25.31.0010, 2017-14-595-0001-003, âĎ́́ 3.9678.2017/8.9, âĎÚ3.904.2017/4.6, 3.6787.2017/7.8, 1.6790.2017/7.8), the Russian Foundation for Basic Research (Grants 16-02-00738, 16-32-00329, 17-02-00905, 16-29-13035) and the grant 15-12-20022 of the Russian Science Foundation (section 2 and 3), and by the German Helmholtz Association and from the European Union's Horizon 2020 programme under grant agreement No. 653477.

\section{References}

[1] S. Wakely, D. Horan, the TeV catalogue, TeVCat, http://tevcat.uchicago.edu/

[2] B.S. Acharya, M. Actis, T. Aghajani, G. Agnetta, J. Aguilar et al., Astr.Part.Phys. 43, 3 (2013)

[3] R. Mirzoyan, et al. (TAIGA collaboration), in 40th COSPAR Sc.Ass (2014), Vol. 40

[4] N. Budnev et al., Journal of Physics: Conference Series 718, 052006 (2016)

[5] M. Tluczykont, D. Hampf, D. Horns et al., Advances in Space Research 48, 1935 (2011)

[6] M. Tluczykont, et al. (TAIGA collaboration), in Connecting Neutrino Physics and Astronomy, edited by S. Dahmke, M. Meyer, L. Vanhoefer (2016), pp. 135-142

[7] M. Tluczykont, D. Hampf, D. Horns, et al., Astroparticle Physics 56, 42 (2014)

[8] O. Gress, et al. (TAIGA collaboration), Nuclear Instruments and Methods: A (2016), http://dx.doi.org/10.1016/j.nima.2016.08.031

[9] A. Porelli, et al. (TAIGA collaboration), J. of Phys: Conf. Ser. 632, 012041 (2015)

[10] S.F. Berezhnev, D. Besson, N.M. Budnev et al., NIMA 692, 98 (2012)

[11] D. Hampf, M. Tluczykont, D. Horns, NIMA 712, 137 (2013)

[12] L. Kuzmichev, et al. (TAIGA collaboration), EPJ Web of Conf. V. 145 (2017) 01001

[13] M. Kunnas, et al. (TAIGA collaboration), J. of Phys.: Conference Series 632, 012040 (2015)

[14] M. Tluczykont, et al. (TAIGA collaboration), J. of Phys.: Conference Series 632, 012042 (2015)

[15] M. Tluczykont, et al. (TAIGA collaboration), Eur. Phys. J. Web of Conf. 136, 03008 (2017)

[16] A. Porelli, et al. (TAIGA collaboration), TAIGA-HiSCORE detection of the CATS-Lidar on the ISS as fast moving point source, These proceedings

[17] A. Porelli, et al., A hybrid time calibration method for EAS ground-based timing arrays, These proceedings

[18] N. Budnev, et al. (TAIGA collaboration), TAIGA - a hybrid detector complex for high energy gamma-ray astro-physics and cosmic ray physics in the Tunka valley, These proceedings

[19] L. Sveshnikova, et al. (TAIGA collaboration), The search for gamma emission above $50 \mathrm{TeV}$ from Crab Nebula in the TAIGA observatory, These proceedings

[20] E. Postnikov, et al. (TAIGA collaboration), Commissioning the joint operation of the wide angle timing HiSCORE Cherenkov array with the first IACT of the TAIGA experiment, These proceedings

[21] http://cats.gsfc.nasa.gov/

[22] http://observ.pereplet.ru/ 